\title{
Effect of the time-weakening friction law during the nucleation process
}

\author{
Feng Hu $\cdot$ Hanqing Huang $\cdot$ Xiaofei Chen
}

Received: 14 February 2016/Accepted: 20 April 2017/Published online: 17 June 2017

(C) The Author(s) 2017. This article is an open access publication

\begin{abstract}
Based on dynamic rupture simulations on a planar fault in a homogeneous half-space, we investigated the nucleation processes using the time-weakening friction law. Both the characteristic time and the rupture speed in the nucleation asperity play an important role in determining rupture behaviors on a fault plane following the time-weakening friction law, with which rupture starts from a single point in the nucleation asperity and propagates at a given speed toward the boundary of the nucleation area. Rupture with a small characteristic time or a large rupture speed in the nucleation asperity propagates earlier from the hypocenter. Rupture following the slipweakening friction law requires a smaller radius of nucleation patch to have similar rupture front contours of the time-weakening friction law. Even if the rupture velocity in the nucleation patch of the time-weakening friction law increases to infinity, the peak slip rate in the nucleation asperity is smaller than that of the slip-weakening law. The peak ground velocity distributions of ruptures following the two friction laws are also compared.
\end{abstract}

Keywords Time-weakening friction law · Slip-weakening friction law $\cdot$ Dynamic rupture $\cdot$ Ground motion

F. Hu $\cdot$ H. Huang

Laboratory of Seismology and Physics of Earth's Interior and School of Earth and Space Sciences, University of Science and Technology of China, Hefei, China

X. Chen $(\bowtie)$

Department of Earth and Space Science, Southern University of Science and Technology, Shenzhen, China

e-mail: xfchen@sustc.edu.cn

\section{Introduction}

The earthquake nucleation process, which is difficult to observe directly in natural earthquakes, is an essential question in dynamic rupture problems. Numerical simulations are usually preferred to investigate the problem. During the spontaneous rupture process, slip starts when the shear traction reaches the friction strength at each point on a given fault following a given constitution law. Both slip-weakening friction law and time-weakening friction law are widely used in rupture dynamics (Andrews 1976; Bizzarri 2010). However, the effect of time-weakening friction law in controlling rupture behaviors during the nucleation process is seldom studied.

Starting from the pioneering work of Ida (1972), and Andrews (1976), the slip-weakening friction law is one of the most adopted constitutive laws in spontaneous rupture simulations (Harris et al. 2009). Although conceptually simple, the corporation of the slip-weakening law and numerical codes can predict the main characteristics of dynamic rupture scenarios on fault systems (Harris et al. 1991; Kase and Kuge 2001; Hu et al. 2016a). The slipweakening behavior has also been noticed using the rateand state-dependent friction law (Cocco and Bizzarri 2002). Compared to the rate- and state-dependent friction law (Dieterich 1979; Ruina 1983), it is easier to accomplish in numerical codes, although a high shear stress patch has to be located on the fault to trigger rupture in the slipweakening friction law.

Thus, the slip-weakening friction law cannot be used to simulate repeated ruptures on a planar fault unless there is external time variable loading inserted (Bizzarri 2010). It is usually assumed that an asperity exists on the fault plane to trigger the rupture, in which the initial shear stress is larger 
than the friction strength. Under this assumption rupture starts simultaneously from all the points in the asperity. There exists another nucleation method referred as the time-weakening friction law (Bizzarri 2010), in which rupture starts from one point in the asperity, and propagates with a given rupture velocity toward the boundary of the nucleation patch. The starting point is treated as the hypocenter. Although Bizzarri (2010) compared the rupture speed dependence on the two nucleation methods, it is still unclear about the factors influencing the time-weakening friction law and equivalence of these two constitution laws in controlling rupture behaviors.

In this paper, we compared dynamic rupture behaviors on a single fault plane in a homogeneous half-space following the time-weakening and slip-weakening friction laws, respectively. The characteristic time and rupture speed in the asperity are two key factors in controlling the rupture fronts following the time-weakening friction law. To have a similar rupture front contour on a planar fault, rupture following the slip-weakening law should have a smaller size of nucleation asperity compared to the result of the time-weakening law. The distributions of peak ground motions are also studied following the two friction laws.

\section{Two different nucleation methods}

The slip-weakening (SW) friction law was first proposed by Ida (1972) and Andrews (1976). Its simplicity in use and accuracy in predicting dynamic rupture behaviors made it widely used in rupture dynamics (Andrews 1985; Madariaga et al. 1998; Zhang and Chen 2006a, b; Hu et al. 2014, 2016b; Xu et al. 2015). As shown in Eq. (1), the fault strength decreases linearly to the residual strength as slip increases from zero to the characteristic slip distance,

$\tau^{(\mathrm{SW})}= \begin{cases}\tau_{u}-\left(\tau_{u}-\tau_{f}\right) u / d_{0}, & u<d_{0} \\ \tau_{f}, & u \geq d_{0}\end{cases}$

where $u$ is the magnitude of the fault slip, $\tau_{u}$ is the fault strength, $\tau_{f}$ is the residual strength, and $d_{0}$ is the characteristic slip distance. However, such a constitution law does not contain any nucleation process. An asperity with a stress perturbation which makes the initial shear stress larger than the friction strength is needed to trigger the rupture. Thus, the fault traction is:

$\tau=\left\{\begin{array}{ll}\tau_{\text {nuc }}=\tau_{0}+\Delta \tau_{\text {nuc }}, & r \leq R_{\text {nuc }} \\ \tau_{0}, & r>R_{\text {nuc }}\end{array}\right.$,

where $\tau_{0}$ is the initial shear stress, $\Delta \tau_{\text {nuc }}$ is the additional shear stress inserted in the asperity to start the rupture. It should be noticed that the time-weakening (TW) friction law here is only used during the nucleation process.
Rupture outside the nucleation patch also follows the SW law. The TW friction is specified as (Bizzarri 2010):

$\tau=\left\{\begin{array}{ll}\tau_{\mathrm{nuc}}=\min \left\{\tau^{(\mathrm{SW})}, \tau^{(\mathrm{TW})}\right\}, & r \leq R_{\mathrm{nuc}} \\ \tau^{(\mathrm{SW})}, & r>R_{\mathrm{nuc}}\end{array}\right.$,

where $\tau^{(\mathrm{SW})}$ is given in Eq. (1) and $\tau^{(\mathrm{TW})}$ is given by,

$\tau^{(\mathrm{TW})}=\left\{\begin{array}{ll}{\left[\mu_{\mathrm{s}}-\left(\mu_{\mathrm{s}}-\mu_{\mathrm{f}}\right)\left(t-t_{\text {force }}\right) / t_{0}\right] \tau_{n},} & t-t_{\text {force }}<t_{0} \\ \mu_{\mathrm{f}} \tau_{n}, & t-t_{\text {force }} \geq t_{0}\end{array}\right.$,

where $\mu_{\mathrm{s}}$ and $\mu_{\mathrm{f}}$ are static and dynamic frictional coefficients, respectively. $t_{0}$ is the characteristic time. $\tau_{n}$ means the normal stress. And

$t_{\text {force }}=\frac{\sqrt{\left(y-y^{H}\right)^{2}+\left(z-z^{H}\right)^{2}}}{v_{\text {force }}}$

where $v_{\text {force }}$ is the rupture speed in the asperity, and $t_{\text {force }}$ is the time at which rupture propagating at $v_{\text {force }}$ reaches the point $(y, z)$ from the hypocenter $\left(y^{H}, z^{H}\right)$. Thus, the fault strength in the asperity decreases linearly as time increases according to the TW law, rather than the slip dependent in the SW law. Slip starts when fault strength decreases to the initial shear stress. Different from the nucleation strategy of the SW law, the initial shear stress using the TW law here is homogeneous through the whole fault plane.

\section{Models and methods}

We construct a right-lateral strike-slip single fault in a homogeneous, isotropic, elastic half-space. The $\mathrm{P}$ wave speed is $6.0 \mathrm{~km} / \mathrm{s}$, and the $\mathrm{S}$ wave speed is $3.464 \mathrm{~km} / \mathrm{s}$. The density is $2670 \mathrm{~kg} / \mathrm{m}^{3}$. As shown in Fig. 1, the fault is

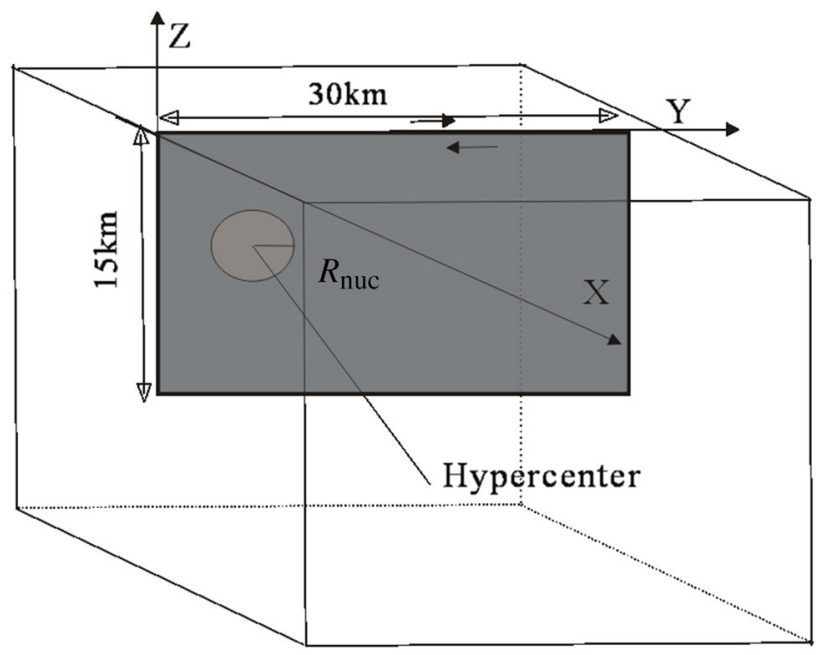

Fig. 1 Illustration of a right-lateral strike-slip fault model in a halfspace 
$30 \mathrm{~km}$ long, $15 \mathrm{~km}$ wide. The hypocenter is located at $\left(y^{H}, z^{H}\right)=(7.5 \mathrm{~km},-7.5 \mathrm{~km})$. The stress and frictional parameters are shown in Table 1. As stated previously, rupture starts simultaneously from all the points in the nucleation asperity following the SW law, but it is triggered from the hypocenter and propagates at a given velocity toward the boundary of nucleation asperity following the TW law. For the SW law, rupture is simultaneously triggered by the higher initial shear stress $\tau_{0}+\Delta \tau_{\text {nuc }}$ (Table 1) in the nucleation asperity. For the two constitution laws, we assume a circle nucleation asperity with a radius of $R_{\text {nuc }}=3 \mathrm{~km}$. The dynamic rupture process of the single fault is calculated using the finite difference method developed by Zhang et al. (2014). The spatial grid is set to be $100 \mathrm{~m}$, and the time interval is $0.01 \mathrm{~s}$.

\section{Rupture behavior controlled by the TW law}

To understand the effects of the characteristic time $t_{0}$ in the TW law, three rupture fronts with different characteristic time $t_{0}$ of $0.3,0.5$ and $0.7 \mathrm{~s}$ are presented in Fig. 2. Rupture speed in the nucleation patch $v_{\text {force }}$ is all set to be $0.75 v_{\mathrm{S}}$ here, where $v_{\mathrm{S}}$ is the $\mathrm{S}$ wave speed. A prominent feature is rupture with a smaller characteristic time propagates earlier. As shown in Eq. (4), a smaller $t_{0}$ means the fault strength in the asperity decreases faster to the residual

Table 1 Stress and frictional parameters

\begin{tabular}{lll}
\hline Parameters & SW law & TW law \\
\hline Initial shear stress $\tau_{0}(\mathrm{MPa})$ & 71.3 & 71.3 \\
Initial normal stress $-\tau_{n}(\mathrm{MPa})$ & 120 & 120 \\
Additional shear stress $\Delta \tau_{\text {nuc }}(\mathrm{MPa})$ & 10.3 & 0 \\
Static friction coefficient $\mu_{\mathrm{s}}$ & 0.677 & 0.677 \\
Dynamic friction coefficient $\mu_{\mathrm{d}}$ & 0.525 & 0.525 \\
Characteristic slip distance $d_{0}(\mathrm{~m})$ & 0.4 & 0.4 \\
\hline
\end{tabular}

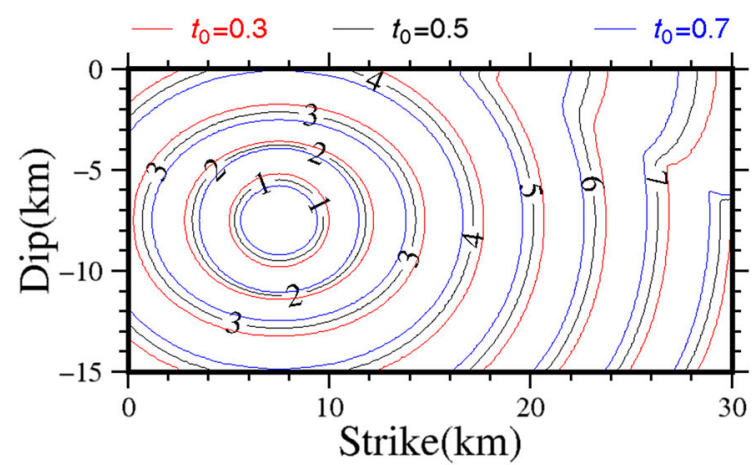

strength. For all the three $t_{0}$ values, free-surface-induced supershear ruptures are all observed. Shortly after $7 \mathrm{~s}$, rupture fronts encounter the end of the fault plane, $30 \mathrm{~km}$ along the strike direction. Figure 2 also shows the horizontal shear stresses at the hypocenter for the three cases. Different from the SW law, the initial shear stress at the hypocenter is $71.3 \mathrm{MPa}$, the same as that outside of the nucleation patch, which is far from the friction strength. As time increases, the friction strength decreases linearly. Slip starts when the friction strength decreases to the initial shear stress. And all the shear stress decreases to the residual stress at the characteristic time $t_{0}$. With a larger $t_{0}$, the shear stress decreases slower.

Different from the effect of characteristic time, a larger $v_{\text {force }}$ means a smaller $t_{\text {force }}$ at the same point on a given fault (Eq. 5). Thus, at the same time, the fault strength in the asperity decreases faster with a larger $v_{\text {force}}$, which permits the rupture to propagate earlier on the fault plane. We compared the space-time plots of horizontal slip velocity along the fault trace $(z=-7.5 \mathrm{~km})$ and the rupture front contours following the TW and SW friction laws, respectively (Fig. 3). Three different values of $v_{\text {force }}$, including $0.75 v_{s}, 1.73 v_{s}$ (which equals to the $P$ wave velocity) and $\infty$ are considered. Although the upper limit of rupture velocity is $\mathrm{P}$ wave velocity, the infinity rupture velocity here considered is only used to study the differences of the rupture behaviors caused by the friction laws themselves, because all the points in the nucleation area following the SW friction law start to rupture simultaneously. The characteristic time $t_{0}$ is set to be $0.5 \mathrm{~s}$ in the three cases following the TW law. However, three different values $R_{\text {nuc }}$ of $1.3,1.5$ and $3.0 \mathrm{~km}$ are considered in the SW law. The plot also gives the $\mathrm{S}$ wave and $\mathrm{P}$ wave arrivals along the fault trace (white dashes).

Two main features are noticed in Fig. 3. First, the rupture velocity in the nucleation patch is identical to the $v_{\text {force }}$. As shown in the space-time slip rate distribution in Fig. 3, when $v_{\text {force }}$ is $0.75 v_{\mathrm{S}}$, rupture propagates at sub-Rayleigh

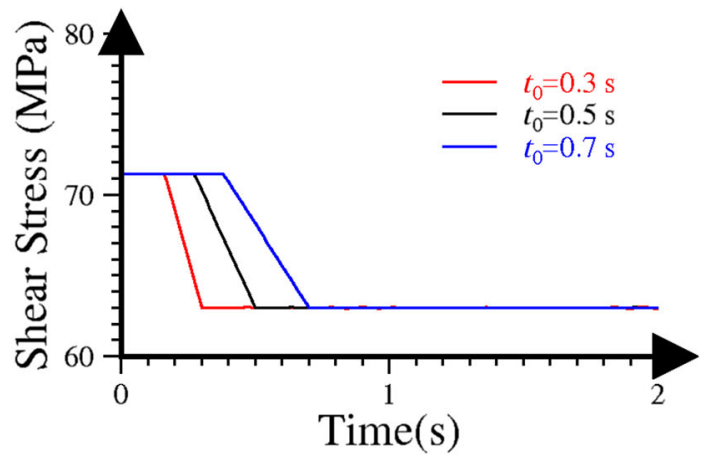

Fig. 2 (left) Rupture front contours of three different characteristic time $t_{0}$ following the TW law. (right) Shear stress versus time at the hypocenter for the three cases 

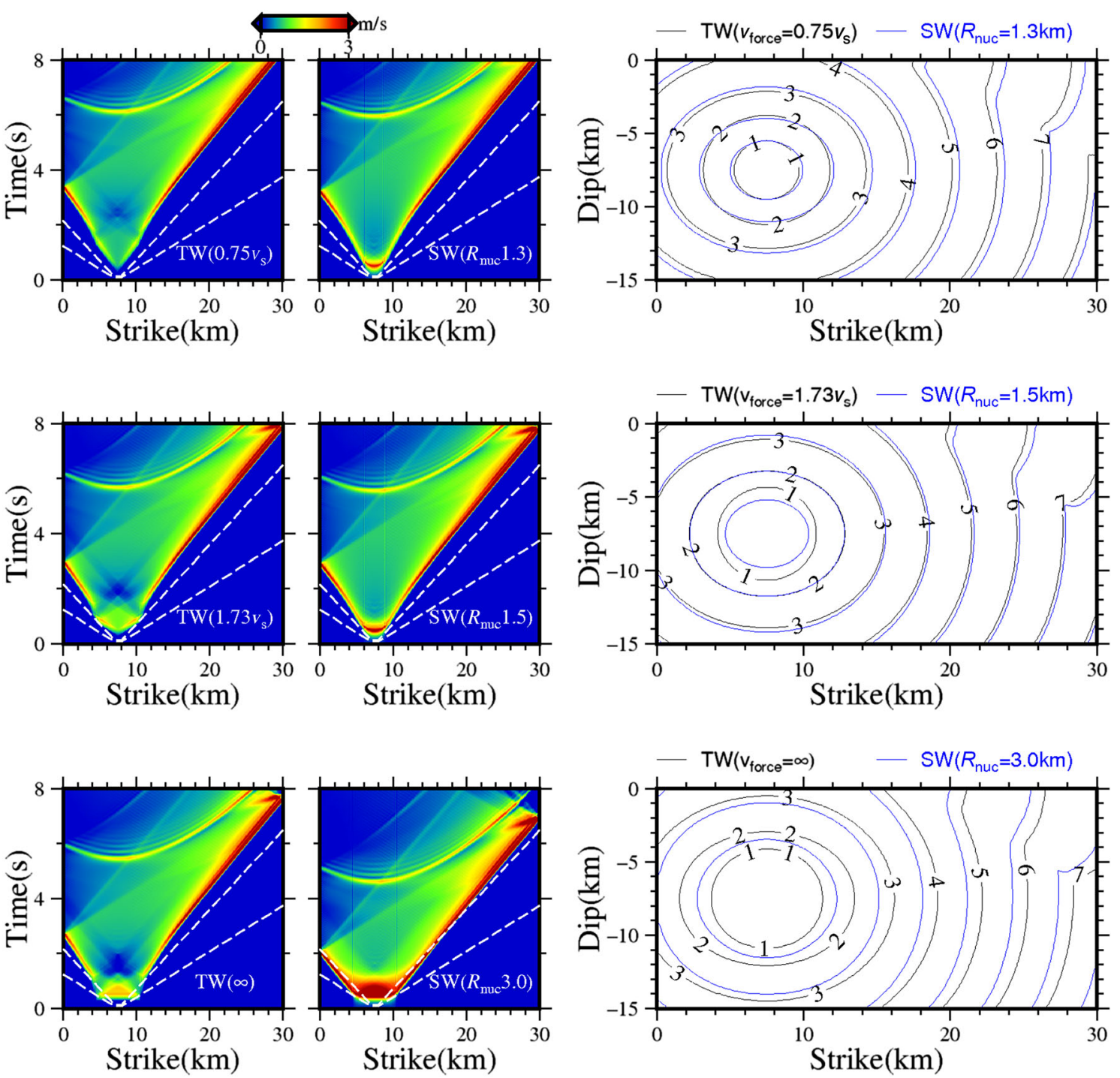

Fig. 3 (left) Space-time plot horizontal slip velocity along the fault trace $(z=-7.5 \mathrm{~km})$. The dashed lines depict wave arrivals along the fault trace from the nucleation patch with three different values of $v_{\text {force }}$ and $R_{\text {nuc }}$ following the TW and SW friction laws, respectively. (right) Rupture front contours comparisons between two different friction laws

speed in the nucleation patch, which increases to $\mathrm{P}$ wave speed when $v_{\text {force }}$ increases to $1.73 v_{\mathrm{S}}$. If $v_{\text {force }}$ is set to $\infty$, all the points in the asperity start to rupture simultaneously, which coincides with the nucleation process of the SW law.

Second, to have similar rupture fronts following the TW laws, rupture following the SW law should have a smaller nucleation radius $R_{\text {nuc }}$. As shown in Fig. 3, rupture following the TW law with a $3 \mathrm{~km} R_{\text {nuc }}$ and $v_{\text {force }}$ of $0.75 v_{\mathrm{S}}$ shows similar characteristics of slip rate on the given fault trace with that following the SW law with $R_{\text {nuc }}$ of $1.3 \mathrm{~km}$. The free-surface-induced supershear has not reached the fault trace in the middle depth at $8 \mathrm{~s}$, which is the same in the slip rate snaps shown in Fig. 3. When the radius of asperity $R_{\text {nuc }}$ increases to $1.5 \mathrm{~km}$ in the SW law, the corresponding rupture following the TW law requires $v_{\text {force }}$ of
$\mathrm{P}$ wave velocity. Nearly at $8 \mathrm{~s}$, the free-surface-induced supershear rupture can be observed in both slip rate snaps. If $R_{\text {nuc }}$ increases to $3 \mathrm{~km}$ in the SW law, the rupture front following the TW law propagates slower than that of the $\mathrm{SW}$ law, even if the $v_{\text {force }}$ is set to $\infty$. As noticed in Fig. 2, the shear stress of the TW law in the asperity decreases from 71.3 to $63.0 \mathrm{MPa}$, instead of 81.6 to $63.0 \mathrm{MPa}$ of the SW law. Thus, the higher slip rate in the asperity is induced by the larger stress drop of the SW law.

An interesting feature is for the case with $v_{\text {force }}$ of $1.73 v_{\mathrm{S}}$, the rupture front travels slightly farther for the TW law at $1 \mathrm{~s}$; however, the rupture front for the SW law travels goes beyond it after $1 \mathrm{~s}$. Such differences may be caused by the transition process from TW to SW law at the boundary of the nucleation asperity (Eq. 3). Different from 
the TW law, stresses at the points just outside of the nucleation asperity have to rise from the initial shear stresses to the static friction strengths of the SW law, which takes some time. This helps to understand the difference between rupture fronts for the TW case with $v_{\text {force }}$ of $\infty$ and the SW case with $R_{\text {nuc }}$ of $3 \mathrm{~km}$.

Another main factor that causes the smaller nucleation size of the SW law with respect to the TW law is the larger peak slip rate in the nucleation asperity. Figure 4 shows the slip rate versus time of the point in the center of the nucleation asperity of the different cases shown in Fig. 3. As the $v_{\text {force }}$ increases in the TW law, the peak slip rate in the hypocenter also increases; however, even if the $v_{\text {force }}$ is set to $\infty$, which is the same in the SW law, the peak slip rate is smaller than that of the $\mathrm{SW}$ law with a $3.0 \mathrm{~km}$ nucleation radius. For all the cases shown in Fig. 4, although their rupture fronts are similar, all peak slip rates of the SW law are larger than those of the TW law, which is caused by the larger stress drop in the SW law. A larger peak slip rate represents a larger fracture energy, which
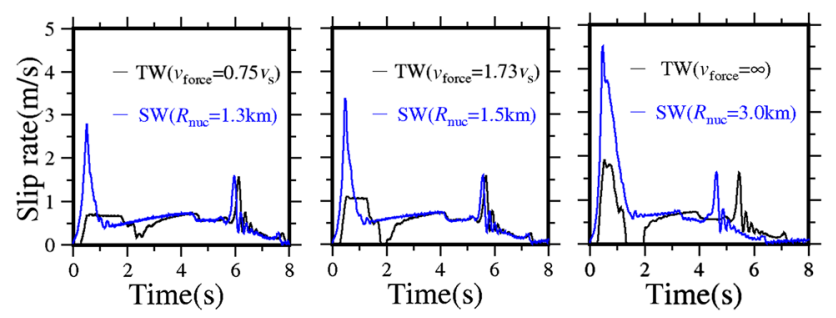

Fig. 4 Slip rate distribution of the single point located at the hypocenter with different cases shown in Fig. 3

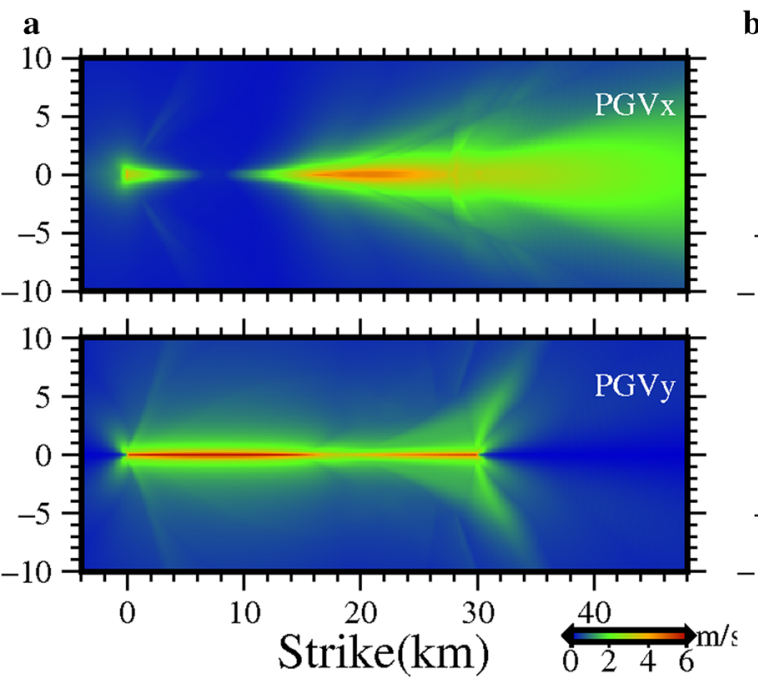

b

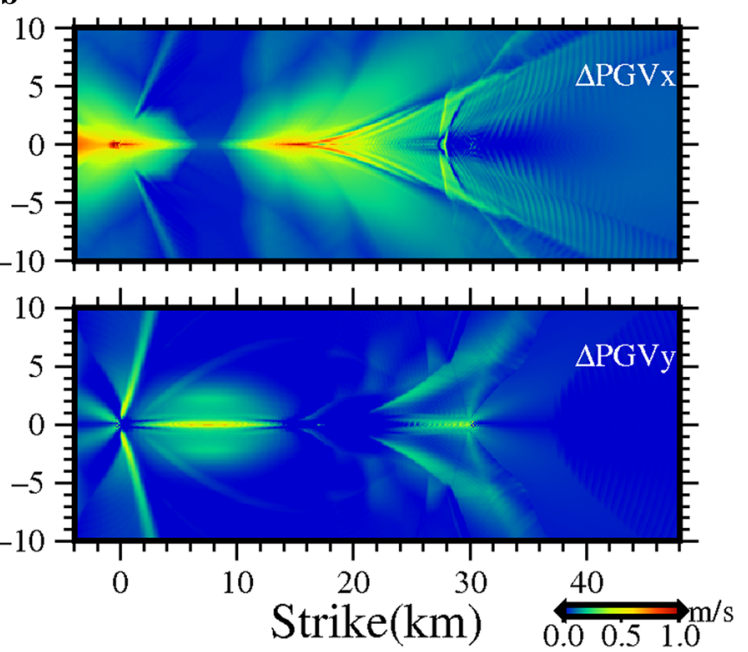

Fig. 5 (left) Distribution of peak ground velocities on the free surface of rupture following the TW law with a $v_{\text {force }}$ of $0.75 v_{\mathrm{S}}$ and a $R_{\text {nuc }}$ of $3 \mathrm{~km}$. (right) Perturbation of peak ground velocities (results of the SW law with a $R_{\text {nuc }}$ of $3 \mathrm{~km}$ minus the result of Fig. 5 (left)) 


\section{Conclusions}

We have performed dynamic rupture simulations using the TW and SW friction laws, respectively, on a planar fault in a homogeneous half-space. Different from the SW law, two factors of the TW law, including the characteristic time $t_{0}$ and the rupture speed in the nucleation asperity $v_{\text {force }}$, control the main characteristics of rupture behaviors on a planar fault. Rupture with a smaller $t_{0}$ or a larger $v_{\text {force }}$ propagates faster on a single fault plane following the TW law.

Rupture fronts following the TW law look similar with those of the SW law with a smaller nucleation asperity. There are two main reasons that cause the phenomenon. The first one is the different nucleation strategies. Rupture of the SW law starts simultaneously in the nucleation patch, while it is triggered in a single point (the hypocenter) in the TW friction law, and propagates at a given speed toward the boundary of the nucleation asperity. According to our simulations, the rupture following the TW law with a $v_{\text {force }}$ of $0.75 v_{\mathrm{S}}$ and a $R_{\text {nuc }}$ of $3 \mathrm{~km}$ has similar rupture front and peak ground velocity distribution with the rupture following the SW law with a $R_{\text {nuc }}$ of $1.3 \mathrm{~km}$. The other factor is that peak slip rates of the TW law are smaller than those of the SW law in the nucleation asperity. Even if the $v_{\text {force }}$ is set to $\infty$, which is the same as that of the SW law, the rupture front also propagates slower than that of the SW law because of the lower slip rate value in the nucleation asperity following the TW friction law.

With the same radius of nucleation asperity, the peak ground velocity distribution near the fault trace caused by the rupture following the TW friction law shows similar characteristics with that of the SW friction law with the same nucleation radius, except the lower effect of rupture directivity and smaller value near the nucleation area in the fault-parallel component. Our work can provide useful insight to understanding the earthquake nucleation process.

Acknowledgements This work is supported by the National Natural Science Foundation of China (Nos. 41504039, 41474037 and 41274053). All data used in this study are derived from numerical simulations.

Open Access This article is distributed under the terms of the Creative Commons Attribution 4.0 International License (http://crea tivecommons.org/licenses/by/4.0/), which permits unrestricted use, distribution, and reproduction in any medium, provided you give appropriate credit to the original author(s) and the source, provide a link to the Creative Commons license, and indicate if changes were made.

\section{References}

Andrews DJ (1976) Rupture velocity of plane strain shear cracks. J Geophys Res 81:5679-5687

Andrews DJ (1985) Dynamic plane-strain shear rupture with a slipweakening friction law calculated by a boundary integral method. Bull Seismol Soc Am 75:1-21

Bizzarri A (2010) How to promote earthquake ruptures: different nucleation strategies in a dynamic model with slip-weakening friction. Bull Seismol Soc Am 100:923-940

Cocco M, Bizzarri A (2002) On the slip-weakening behavior of rateand state dependent constitutive laws. Geophys Res Lett 29(11): 11

Dieterich JH (1979) Modeling of rock friction: 1. Experimental results and constitutive equations. J Geophys Res 84:2161

Harris RA, Archuleta RJ, Day SM (1991) Fault steps and the dynamic rupture process: 2-D numerical simulations of a spontaneously propagating shear fracture. Geophys Res Lett 18:893-896

Harris RA, Barall M, Archuleta R, Dunham E, Aagaard B, Ampuero JP, Bhat H, Cruz-Atienza V, Dalguer L, Dawson P, Day S, Duan B, Ely G, Kaneko Y, Kase Y, Lapusta N, Liu Y, Ma S, Oglesby D, Olsen K, Pitarka A, Song S, Templeton E (2009) The SCEC/ USGS dynamic earthquake rupture code verification exercise. Seismol Res Lett 80:119-126

Hu F, Xu J, Zhang Z, Zhang W, Chen X (2014) Construction of equivalent single planar fault model for strike-slip stepovers. Tectonophysics 632:244-249

Hu F, Zhang Z, Chen X (2016a) Investigation of earthquake jump distance for strike-slip step overs based on 3-D dynamic rupture simulations in an elastic half-space. J Geophys Res 121:994-1006

Hu F, Xu J, Zhang Z, Chen X (2016b) Supershear transition mechanism induced by step over geometry. J Geophys Res. doi:10.1002/2016JB013333

Ida Y (1972) Cohesive force across the tip of a longitudinal-shear crack and Griffith's specific surface energy. J Geophys Res 77:3796-3805

Kase Y, Kuge K (2001) Rupture propagation beyond fault discontinuities: significance of fault strike and location. Geophys J Int 147:330-342

Madariaga R, Olsen K, Archuleta R (1998) Modeling dynamic rupture in a 3D earthquake fault model. Bull Seismol Soc Am $88: 1182-1197$

Ruina A (1983) Slip instability and state variable friction laws. J Geophys Res 88(B12):10359-10370

Xu J, Zhang H, Chen X (2015) Rupture phase diagrams for a planar fault in 3-D full-space and half-space. Geophys $\mathrm{J}$ Int 202:2194-2206

Zhang H, Chen X (2006a) Dynamic rupture on a planar fault in threedimensional half space-I. Theory. Geophys. J. Int. 164:633-652

Zhang H, Chen X (2006b) Dynamic rupture on a planar fault in threedimensional half-space-II. Validations and numerical experiments. Geophys J Int 167:917-932

Zhang Z, Zhang W, Chen X (2014) Three-dimensional curved grid finite-difference modelling for non-planar rupture dynamics. Geophys J Int 199:860-879 